\title{
The Antibiotic-Vaseline Soaked Cotton Pledget as an Adjuvant Material for Endoscopic Endonasal Fungal Ball Removal
}

\author{
Young Yoon Kim, $\mathrm{MD}^{1}$, Hyung Chae Yang, $\mathrm{MD}, \mathrm{PhD}^{1}$, Jae Gu Kim, $\mathrm{MD}^{1}$, Hee Young Kim, $\mathrm{MD}^{1}$, \\ Jong Min Park, MD ${ }^{1}$, Kwang Il Nam, MD, $\mathrm{PhD}^{2}$, Min-Keun Song, $\mathrm{MD}, \mathrm{PhD}^{3}$, and Sang Chul Lim, $\mathrm{MD} \mathrm{PhD}^{1}$ \\ ${ }^{1}$ Department of Otolaryngology-Head and Neck Surgery, Chonnam National University Medical School and Chonnam National University \\ Hospital, Gwangju; and ${ }^{2}$ Department of Anatomy, Chonnam National University Medical School, Gwangju; and \\ ${ }^{3}$ Department of Physical and Rehabilitation Medicine, Chonnam National University Medical School and Chonnam National University \\ Hospital, Gwangju, Korea
}

Background and Objectives: Treatment choice for fungal ball is endoscopic endonasal removal. However, it is not easy to remove fungal elements from the maxilla using only an endonasal approach. To overcome this difficulty, we introduced a cotton pledget technique and evaluated its efficacy through a cadaveric study and clinical research.

Materials and Method: A cadaveric study was performed using 10 half heads of seven cadavers. The ease and safety of the cotton pledget technique were compared to those of a previously reported technique. In clinical research, we enrolled 52 patients who underwent surgery with the cotton pledget technique and 36 patients who underwent surgery using the conventional endoscopic approach. Demographic factors, preoperative Lund-Mackay (LM) score, sinonasal outcome test (SNOT) score, surgical morbidity, and incomplete removal rate were analyzed.

Results: The cadaveric study showed that the cotton pledget technique was easier $(p=0.011)$ than the conventional technique. In addition, clinical evaluation showed that the cotton pledget group had significantly lower incomplete removal rate than that of the control group $(\mathrm{p}=0.010)$.

Conclusion: The cotton pledget technique is an easy and safe method that enables fungal ball removal more effectively than the conventional technique without need for inferior meatal antrostomy (IMA) or the Caldwell-Luc (CL) approach.

KEY WORDS: Sinusitis · Mycetomas · Endoscopy $\cdot$ Paranasal sinuses $\cdot$ Minimally invasive surgical procedure $\cdot$ Maxillay.

\section{INTRODUCTION}

Fungal ball is non-invasive mass-like material commonly found in elderly population. ${ }^{1)}$ Different from invasive form of fungal infection, fungal ball is more prevalent in immune-competent patients. ${ }^{2}$ The treatment of choice is surgical removal without using antifungal agent. However, the development of the maxillary sinus differs depending on the individual, the maxillary sinus volume and the depth of the alveolar recess may be different. ${ }^{3)}$ Therefore, if a fungal element was impacted in the deep alveolar recess, it is not easy to remove completely. In this case, inferior meatal antrostomy (IMA), Caldwell-Luc operation (CL-op) with gingivobuccal incision, and or sinus irrigation will be selectively performed, depending on the surgeon.

However, such additional procedures may lead to more comorbidities. IMA may cause unnatural drainage pathway. ${ }^{45)} \mathrm{CL}$-op might cause facial swelling, gingival numbness, decreased facial sensation, wound infection, fibrosis, and abnormal bone changes due to gingivobuccal incision. As a result, CL-op has been replaced by endoscopic endonasal approach in many surgical fields. ${ }^{6-9)}$ In addition, Sinus

Received: March 11, 2019 / Revised: July 8, 2019 / Accepted: August 8, 2019

Address for correspondence: Hyung Chae Yang, MD, PhD, Department of Otolaryngology-Head and Neck Surgery, Chonnam National University Medical School and Chonnam National University Hospital, 42 Jebong-ro, Dong-gu, Gwangju 61469, Korea

Tel: +82-62-220-6776, Fax: +82-62-228-7743, E-mail: blessed@jnu.ac.kr 
irrigations were frequently insufficient for complete removal of the impacted fungal material and may lead to fungal migration.

In this study, antibiotic-vaseline soaked cotton pledget was introduced as an adjuvant material for endoscopic endonasal mycetoma removal to avoid IMA or CL-op. The clinical efficacy of cotton pledget techniques and cadaveric evaluation for their easiness and safety was performed.

\section{MATERIALS AND METHODS}

\section{Cadaver dissection}

Cadaveric studies were performed to evaluate the feasibility of cotton pledget technique. In addition, we compared cotton pledget technique with previously reported saline gauze technique. Two techniques were similar in their surgical technique. However, adjuvant material used in each technique was different. Antibiotic-vaseline soaked cotton pledget was proposed in this study, and saline soaked gauze previously introduced by Chao and Liu. ${ }^{10)}$ Both adjuvant materials were compared in terms of easiness and safety.

A total of 10 half heads from 7 cadavers were used in this study. The mean age of donors was $73.8 \pm 16.0$ years. These cadavers were of Korean descent. They were bequeathed to a university hospital (for blind review) and preserved by anatomical embalming using formalin. This study was approved by the Medical Research Ethics Committee of a university hospital (for blind review). This study adhered to principles defined in the Declaration of Helsinki (1964).

After made a middle meatus antrostomy (MMA), simulation was continued with axially cut cadaver heads to confirm the exact surgical procedure and thoroughly evaluate mucosal injury for paired comparison of cotton pledget and gauze. It was initiated after placing the bulk of adjuvant material in the middle meatus. The adjuvant material was then pushed antero-latero-inferiorly using ball-tip curved suction or 90-degree giraffe forceps (Fig. 1). The procedure was continued until the bulk fully touched the alveolar recess of maxillary sinus. If the surgeon pushes the bulk of the gauze laterally and inferiorly as proven in previous reports, the fungal material will move out toward the maxillary antrostomy site. ${ }^{1011)}$ The same procedure was performed with two techniques in random sequence: antibiotic-vaseline soaked cotton pledget and saline soaked gauze.

Easiness in surgical removal and degree of mucosal injury were evaluated by a junior surgeon who was not an au- thor of this article. The easiness of placing adjuvant material into the alveolar recess of maxillary sinus was evaluated using a $10-\mathrm{cm}$ visual analogue scale (VAS). In addition, longest diameters of mucosal tears were summed to evaluate mucosal trauma. The purpose of such evaluation was to find easier and less traumatic adjuvant material for endoscopic fungal ball removal in patients.

\section{Patients}

Clinical analysis was focused on the evaluation of efficacy of using cotton pledget technique. Retrospective chart reviews of 88 patients with fungal sinusitis were performed. All 88 patients underwent endoscopic sinus surgery from August 2010 to July 2016 in a tertiary hospital. Fifty-two patients who underwent fungal ball removal using cotton pledget technique were assigned into 'cotton pledget group'. On the other hand, 36 patients who underwent fungal ball removal without adjuvant material were assigned into the control group. Demographic factors, underlying diseases, preoperative Lund-Mackay (LM) scores, follow-up durations, type of surgeries, types of anesthesia, and incomplete removal rates of both groups were analyzed. All procedures performed in studies involving human participants were in accordance with the ethical standards of the institutional and/or national research committee and with the 1964 Helsinki declaration and its later amendments or comparable ethical standards (IRB number: CNUH2017-198).

\section{Surgical methods for conventional fungal ball removal}

Surgery was performed under local anesthesia or general anesthesia. Gauze strip soaked with a mixture of $4 \%$ lidocaine and 1:100000 epinephrine for reducing mucosal swelling was inserted through middle meatus. Sphenopalatine ganglion and anterior ethmoid block were performed with $1 \%$ lidocaine with 1:100000 epinephrine with $23 \mathrm{G}$ needle.

To provide transnasal corridor to fungal material, an endoscopic middle meatal antrostomy (MMA) was made following the conventional endoscopic sinus surgery technique. ${ }^{12)}$ An uncinectomy was performed using a sickle knife or a cottle elevator. The natural ostium of maxillary sinus was enlarged and fungal material was identified using $30^{\circ}$ and $70^{\circ}$ endoscopy. After adequate antrostomy was made, fungal material was removed via MMA using ball-tip 

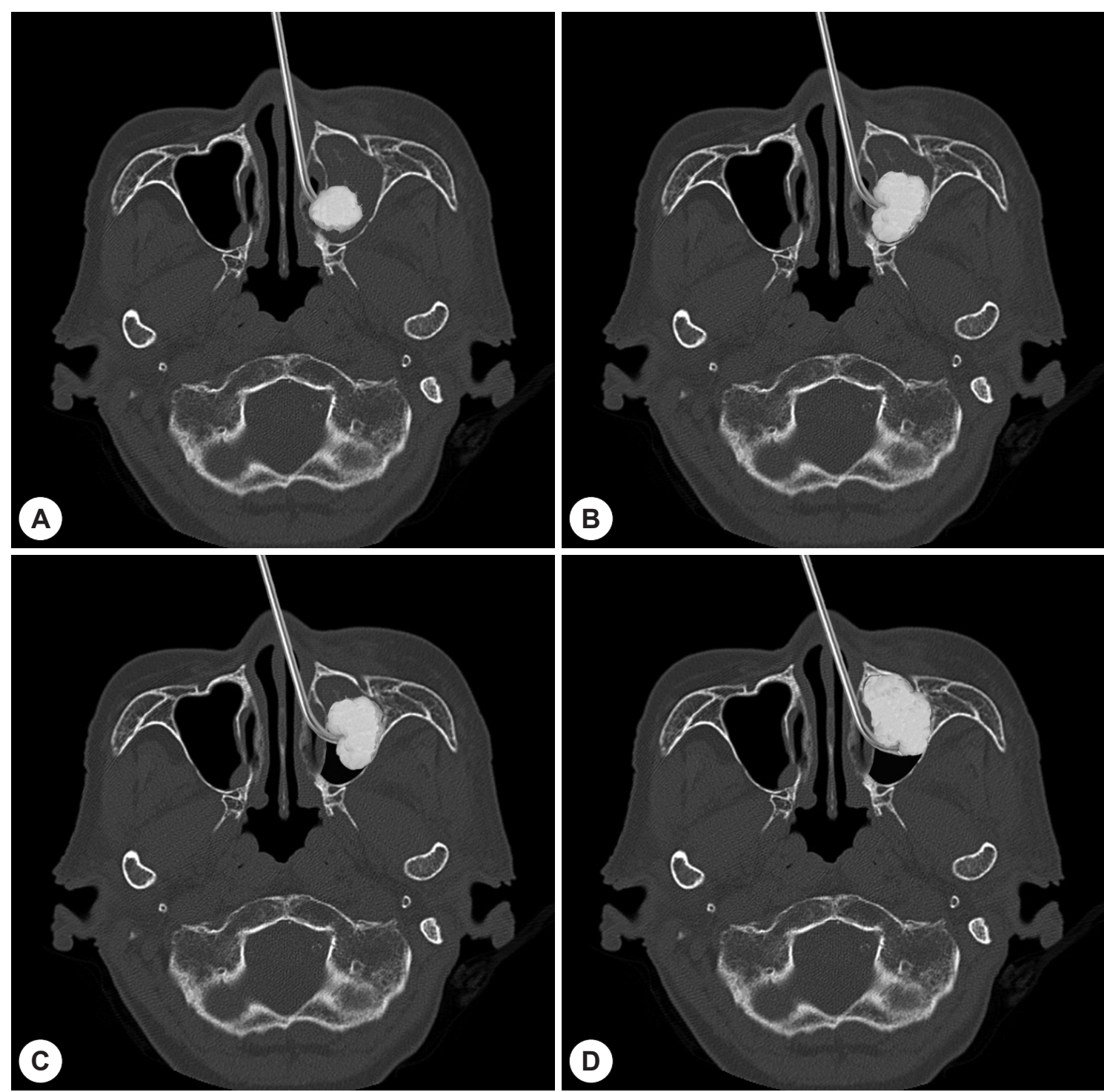

Fig. 1. Cadaveric feasibility study of endonasal fungal ball removal. Antibiotic-vaseline soaked cotton pledget was used as an adjuvant material. A: Vaseline coated cotton pledget holding with forceps. And packed cotton pledget formed a ball-shaped bulk with $90^{\circ}$ frontal sinus recess giraffe forceps. B: The cadaver head was then cut at the level of the middle meatus to visualize the procedure. C: Without cotton pledget, the alveolar recess of maxillary sinus cannot be pushed. D: When cotton pledget was introduced to the maxillary sinus, a surgeon could push the alveolar recess of maxillary sinus.

curved suction or 90-degree giraffe forceps in both groups. Patients with antral puncture were excluded from this study.

\section{Surgical methods for cotton pledget group}

Vaseline coated cotton pledgets were packed at the antrostomy site (Fig. 2, 3). Using ball-tip curved suction, packed adjuvant material into the nasal cavity. In the nasal cavity, the packed adjuvant material usually formed a ball-shaped bulk. As the ball-shaped cotton bulk occupying the maxillary sinus, cheese-like fungal material spilled out from the maxillary sinus to the middle meatus via the antrostomy site. If it was not enough, the operator pushed the adjuvant material anterolaterally along the lateral wall of maxillary sinus using $90^{\circ}$ frontal sinus recess giraffe forceps (Fig. 1). The remnant fungal material was then pushed out to the middle meatus

\section{Statistical analysis}

All values are expressed as numbers or means \pm SD. In cadaveric study, significant differences between the gauze group and the cotton pledget group were determined with Wilcoxon signed ranked test. In clinical study, significant differences between the control group and the cotton pledget group were determined with Fisher's exact test or Stu- 

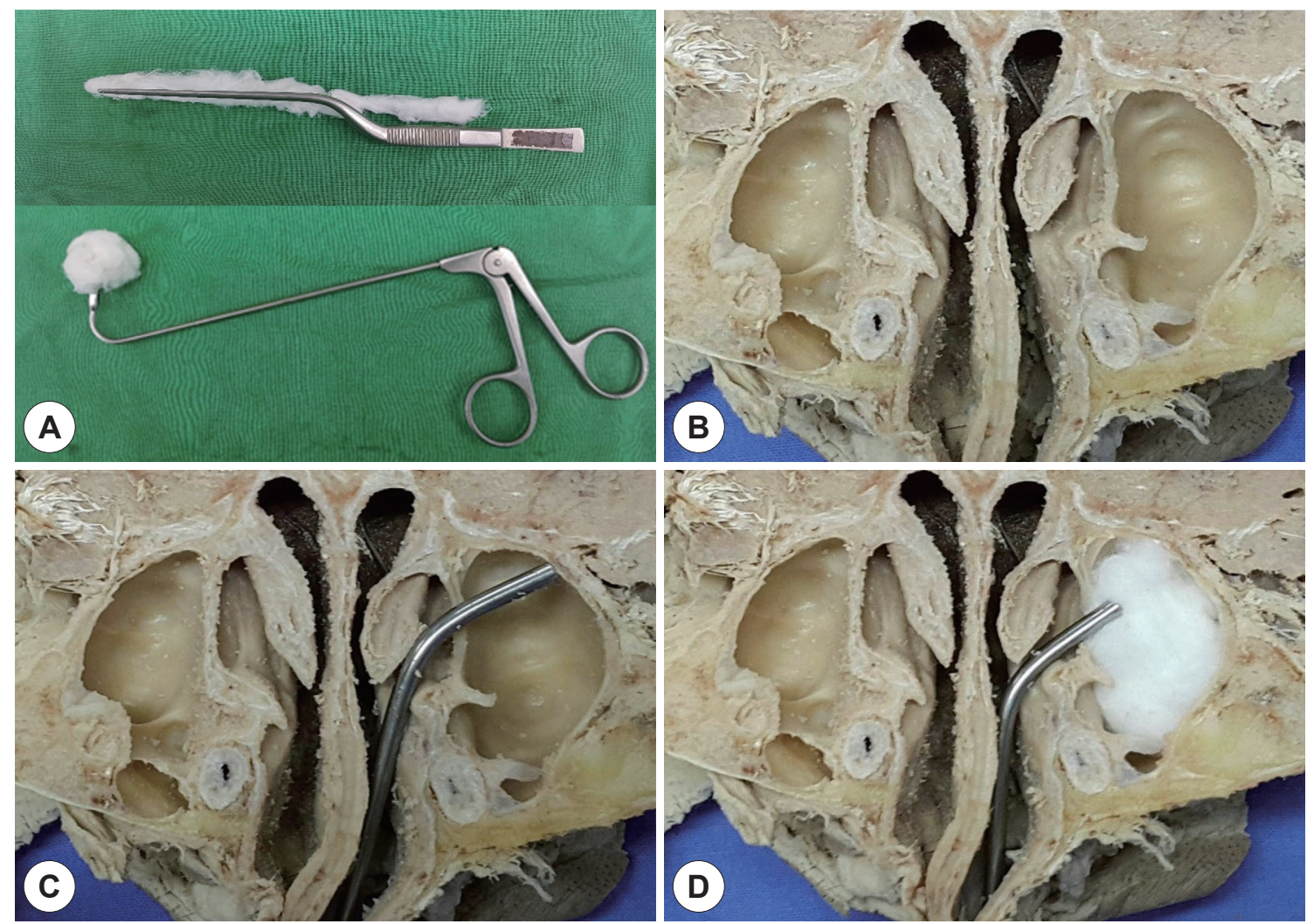

Fig. 2. Phases of fungal ball removal. Cotton pledget was placed in the middle meatus (A) and introduced into the maxillary sinus (B). The maxillary sinus was loosely packed with cotton pledget (C). When pushing the packed cotton pledget into the maxillary sinus, it pushed the fungal material of alveolar recess toward the maxillary sinus ostium (D).

dent's t-test. Statistical significance was set at $\mathrm{p}<0.05$.

\section{RESULTS}

\section{Cadaveric study}

Mean VAS scores for easiness of gauze and cotton pledget as adjuvant materials were $3.20 \pm 1.03$ and $4.90 \pm 1.37$, respectively, with cotton pledget showing significantly $(\mathrm{p}=$ 0.011 ) higher easiness (Table 1). Sizes of mucosal tears in gauze and cotton pledget groups were $3.50 \pm 5.06$ and $0.50 \pm$ 1.58 , respectively. However, the difference of mucosa tears size between the two groups was not statistically significant $(\mathrm{p}=0.068)$.

\section{Clinical study}

Mean age of the study population was $65.64 \pm 10.80$ years. Gender, age, prevalence of hypertension and diabetes, site of disease, or preoperative Lund-Mackay score was not significantly different between the control group and the adjuvant group (Table 2). There were no significant differences in follow up duration or types of surgery between the groups either. However, the adjuvant group showed significantly $(p=0.010)$ lower incomplete removal rate or remnant fungal material than the control group (Table 1).

\section{DISCUSSION}

Fungal ball is non-invasive mass-like material commonly found in the elderly. ${ }^{13)}$ It is more prevalent in hosts with normal immune response. Signs and symptoms of fungal balls are often non-specific and indolent in early stage. ${ }^{14)}$ The most common symptoms are purulent nasal discharges and nasal obstructions often accompanied by polyp or sinusitis. ${ }^{15) 16)}$ Lesions of cheese-like material or mucopurulent discharge in maxillary sinus during surgery may also occur. ${ }^{17)}$

Diagnosis of fungal ball can be done by computed tomography $(\mathrm{CT})$ based on characteristics such as increased shading, micro-calcifications, heterogeneous opacifications, and metal-dense spot of the sinus. ${ }^{717)}$ Magnetic resonance images (MRI) can also be used for its diagnosis. T2 MRI images of the fungal mass show marked hypo-intense signal due to iron, magnesium, manganese, and calcium. There- 

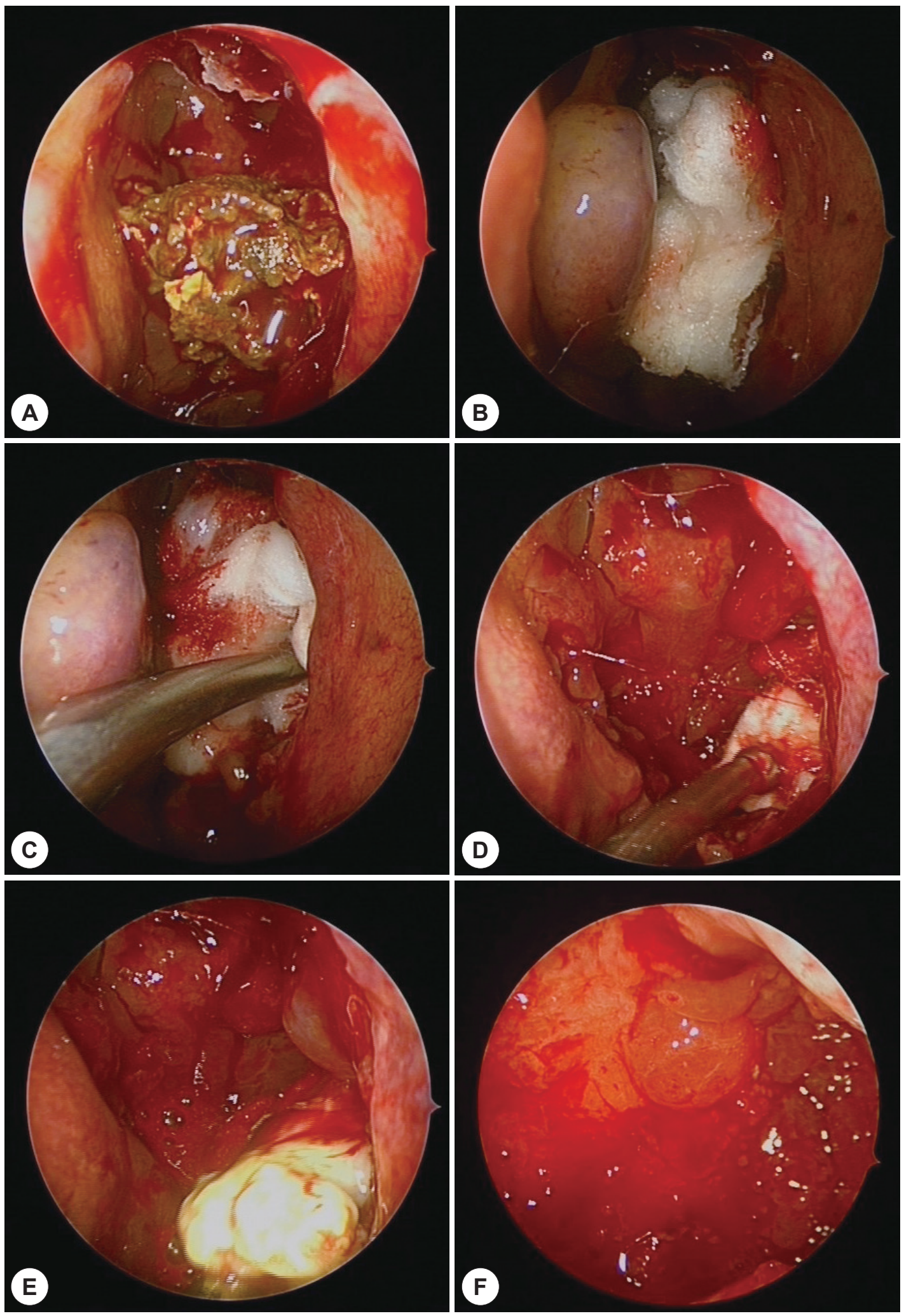

Fig. 3. Phases of endoscopic fungal ball removal. The fungal ball appeared as maxillary sinus ostium after middle meatus antrostomy (A). Cotton pledget was placed in the middle meatus (B) and introduced into the maxillary sinus (C). When pushing the packed cotton pledget into the maxillary sinus, it pushed the fungal material of alveolar recess toward the maxillary sinus ostium (D). Cheese-like fungal material spilled out from the maxillary sinus to the middle meatus via the antrostomy site (E). The postoperative image of the maxillary sinus showed that the fungal material was completely removed (F). 
fore, fungal ball has more characteristic features on MRI than that on $\mathrm{CT}^{18)}$

Treatment of choice for fungal sinusitis is surgical removal without using antifungal agents. However, there is controversy regarding surgery techniques. Before the advent of endoscopic surgical technique for sinus disease (ESS), Caldwell-Luc operation was the mainstay for fungal removal. However, after the advent of ESS technique, endoscopic approaches for fungal sinusitis has gained more popularity than Caldwell-Luc operation. ${ }^{19)}$ Costa el al. have suggested that endoscopic MMA is the gold standard for fungal ball while a Caldwell-Luc approach has to be reserved for selected cases such as fungal ball of alveolar recess of maxillary sinus. ${ }^{19)}$ Although endoscopic approach is a good method, it is hard to remove fungal materials of alveolar recess of maxillary sinus by endoscopic approach. Additional antral punctures are performed frequently. However, antral puncture is not a perfect solution in terms of its morbidity. ${ }^{45)}$ It could cause facial swelling, gingival numbness, decreased facial sensation, and wound infection. ${ }^{4)}$

In this study, we proposed antibiotic-vaseline soaked cotton pledget as an adjuvant material for fungal ball removal instead of antral puncture. Saline soaked gauzes could also be used as adjuvant material. ${ }^{10)}$ Saline soaked gauze technique was a great idea. We also had experience in using

Table 1. Comparative cadaveric study of fungal ball removal techniques

\begin{tabular}{lccc}
\hline & $\begin{array}{c}\text { Gauze } \\
\text { technique }\end{array}$ & $\begin{array}{c}\text { Cotton pledget } \\
\text { technique }\end{array}$ & p-value \\
\hline Number of subjects & 10 & 10 & \\
Easiness (VAS 1-10) & $3.20 \pm 1.03$ & $4.90 \pm 1.37$ & 0.011 \\
Mucosal injury (mm) & $3.50 \pm 5.06$ & $0.50 \pm 1.58$ & 0.068
\end{tabular}

$*$ : Wilcoxon signed ranked tests were used for the analysis of easiness and mucosal injury caused by each technique gauze as an adjuvant material for endoscopic maxillary sinus wall reduction. ${ }^{8)}$ It is a great material for force delivery and stability. However, we thought that the movement of vaseline-coated cotton could be smoother and softer than gauze in the maxillary sinus. Therefore, comparative cadaveric study was performed to select proper adjuvant material. Cadaveric study showed the superiority of vaseline-coated cotton pledget in term of easiness (Table 1). In addition, the degree of mucosal damage caused by cotton pledget was less than that caused by gauze. Although, the different between the two was not statistically significant (Table 1), we should take into account that a small number of dissected cadavers were used in the present study.

We used antibiotic-vaseline coated cotton pledget instead of performing antral puncture in this study. Antibiotic-vaseline coated cotton pledget allowed gentle detachment of cheese-like fungal materials from the alveolar recess of maxillary sinus. Its treatment result of the cotton pledget group was significantly better than that of the conventional gauze group. Four patients in the cotton pledget group had remnant fungal material. Three out of these four cases revealed a small amount of remnant fungal material in the early postoperative period within two weeks. All fungal materials in these cases were already detached from maxillary sinuses and easily removed by using a curved suction tip at our outpatient department (OPD). On the contrary, remnant fungal debris in the control group could not be removed easily. All five cases required reoperations.

Although this study has a limitation due to its retrospective, nonrandomized, and non-placebo-controlled study design, our study results showed that fungal ball removal using antibiotic-vaseline soaked cotton pledget was not only easier and time-saving but also an effective in terms of com-

Table 2. Clinical characteristic of patients with fungal sinusitis according to fungal ball removal technique

\begin{tabular}{lccc}
\hline & Control & Cotton pledget & p-value \\
Number of subjects & 36 & 52 & 0.284 \\
Gender (m:f) & $13: 23$ & $25: 27$ & 0.760 \\
Age (mean \pm SD) & $65.31 \pm 10.52$ & $64.58 \pm 11.23$ & 0.829 \\
Hypertension & 17 & 27 & 0.284 \\
Diabetes & 9 & 8 & 1.000 \\
Anesthesia (G:L) & $0: 36$ & $1: 51$ & 0.674 \\
Preoperative LM score & $3.62 \pm 1.33$ & $3.79 \pm 1.32$ & 0.324 \\
Follow up duration (months) & $7.75 \pm 9.21$ & $6.18 \pm 5.59$ & 0.010 \\
Remnant & $5(13.8 \%)$ & $0(0 \%)$ & \\
\hline
\end{tabular}

Chi-square and Fisher's exact tests were used for the analysis of gender, underlying disease, site of disease, and anesthesia. Student's t-test was used for the analysis of age and preoperative LM score. M: male, F: female, SD: standard deviation, G: General, L: Local, LM: Lund-Mackey 
plete removal compared to that using the conventional fungal ball removal procedure.

\section{CONCLUSION}

The cotton pledget technique enables to remove fungal ball more effectively without using IMA or CL approach. In addition, it is more easy and less traumatic then previously suggested techniques.

\section{Acknowledgments}

This research was supported by Basic Science Research Program through the National Research Foundation of Korea (NRF) funded by the Ministry of Education(NRF-2018R1D1A1B07046906) and a grant (BCRI-18020) from Chonnam National University Hospital Biomedical Research Institute.

\section{Author Contributions}

Analysis and interpretation of data, Drafting of the manuscript, Revision of the manuscript: Young Yoon Kim

Study concept and design, Drafting of the manuscript, Revision of the manuscript, Statistical analysis, Study supervision: Hyung Chae Yang

Acquisition of data: Jae Gu Kim

Acquisition of data: Hee Young Kim

Analysis and interpretation of data: Jong Min Park

Analysis and interpretation of data, Revision of the manuscript: Kwang Il Nam

Study concept and design: Min-Keun Song

Study supervision: Sang Chul Lim

\section{REFERENCES}

1) Dufour X, Kauffmann-Lacroix C, Ferrie JC, Goujon JM, Rodier MH, Karkas A, et al. Paranasal sinus fungus ball and surgery: a review of 175 cases. Rhinology 2005;43(1):34-9.

2) Jiang RS, Hsu CY. Serum immunoglobulins and IgG subclass levels in sinus mycetoma. Otolaryngol Head Neck Surg 2004;130(5): 563-6.

3) Butaric LN. Differential Scaling Patterns in Maxillary Sinus Volume and Nasal Cavity Breadth Among Modern Humans. Anat Rec (Hoboken) 2015;298(10):1710-21.

4) Penttila MA, Rautiainen ME, Pukander JS, Karma PH. Endoscopic versus Caldwell-Luc approach in chronic maxillary sinusitis: com- parison of symptoms at one-year follow-up. Rhinology 1994;32(4): 161-5.

5) Costa F, Polini F, Zerman N, Robiony M, Toro C, Politi M. Surgical treatment of Aspergillus mycetomas of the maxillary sinus: Review of the literature. Oral Surgery, Oral Medicine, Oral Pathology, Oral Radiology, and Endodontology 2007;103(6):e23-9.

6) Costa F, Polini F, Zerman N, Sembronio S, Toro C, Politi M. Functional endoscopic sinus surgery for the treatment of Aspergillus mycetomas of the maxillary sinus. Minerva Stomatol 2008;57(3):117-25.

7) Klossek JM, Serrano E, Peloquin L, Percodani J, Fontanel JP, Pessey JJ. Functional endoscopic sinus surgery and 109 mycetomas of paranasal sinuses. Laryngoscope 1997;107(1):112-7.

8) Yang HC, Kim YY, Jin SM, You H, Han JH, Nam KI. Transnasal endoscopic ultrasound-guided reduction of maxillary sinus wall fracture. Eur Arch Otorhinolaryngol 2017;274(9):3383-90.

9) Yang HC, Kang SH, Yoon SH, Cho HH. Transnasal endoscopic removal of bilateral postoperative maxillary cysts after aesthetic orthognathic ssurgery: Differences from that of Caldwell-Luc operations. Auris Nasus Larynx 2018;45(3):608-12.

10) Chao TK, Liu CM. Gauze-assisted technique in endoscopic removal of fungus balls of the maxillary sinus. Am J Rhinol 2006;20(4): 417-20.

11) Garofalo P, Griffa A, Dumas G, Perottino F. "Gauze Technique" in the Treatment of the Fungus Ball of the Maxillary Sinus: A Technique as Simple as It Is Effective. Int J Otolaryngol 2016;2016:4169523.

12) Kennedy DW, Zinreich SJ, Shaalan H, Kuhn F, Naclerio R, Loch E. Endoscopic middle meatal antrostomy: theory, technique, and patency. Laryngoscope 1987;97(8 Pt 3 Suppl 43):1-9.

13) Shams MG, Motamedi MH. Aspergilloma of the maxillary sinus complicating an oroantral fistula. Oral Surg Oral Med Oral Pathol Oral Radiol Endod 2003;96(1):3-5.

14) Thery A, Espitalier F, Cassagnau E, Durand N, Malard O. Clinical features and outcome of sphenoid sinus aspergillosis: A retrospective series of 15 cases. Eur Ann Otorhinolaryngol Head Neck Dis 2012;129(4):179-84.

15) Campbell MJ, Clayton YM. Bronchopulmonary aspergillosis. a correlation of the clinical and laboratory findings in 272 patients investigated for bronchopulmonary aspergillosis. Am Rev Respir Dis 1964; 89:186-96.

16) Dufour X, Kauffmann-Lacroix C, Ferrie JC, Goujon JM, Rodier MH, Klossek JM. Paranasal sinus fungus ball: epidemiology, clinical features and diagnosis. A retrospective analysis of 173 cases from a single medical center in France, 1989-2002. Med Mycol 2006; 44(1):61-7.

17) deShazo RD, O’Brien M, Chapin K, Soto-Aguilar M, Swain R, Lyons $\mathrm{M}$, et al. Criteria for the diagnosis of sinus mycetoma. J Allergy Clin Immunol 1997;99(4):475-85.

18) Zinreich SJ, Kennedy DW, Malat J, Curtin HD, Epstein JI, Huff LC, et al. Fungal sinusitis: diagnosis with $\mathrm{CT}$ and MR imaging. Radiology 1988;169(2):439-44.

19) Costa F, Polini F, Zerman N, Robiony M, Toro C, Politi M. Surgical treatment of Aspergillus mycetomas of the maxillary sinus: review of the literature. Oral Surg Oral Med Oral Pathol Oral Radiol Endod 2007;103(6):e23-9. 